International Journal of Pure and Applied Mathematics

Volume 87 No. $1 \quad 2013,81-94$

ISSN: 1311-8080 (printed version); ISSN: 1314-3395 (on-line version)

url: http://www.ijpam.eu

doi: http://dx.doi.org/10.12732/ijpam.v87i1.4

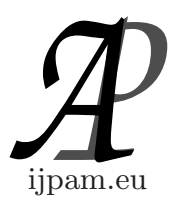

\title{
THE RELATIONSHIP BETWEEN QUASI-MEDIAL IDEMPOTENTS AND QUASI-ADEQUATE TRANSVERSALS
}

\author{
Xiangfei $\mathrm{Ni}$ \\ Department of Mathematics \\ Zhejiang Normal University \\ Jinhua, Zhejiang, 321004, P.R. CHINA
}

\begin{abstract}
Some properties for an abundant semigroup with quasi-medial idempotents are obtained and then the relationship between quasi-medial idempotents and quasi-adequate transversal is characterized.
\end{abstract}

AMS Subject Classification: 20M10

Key Words: abundant semigroup, quasi-medial idempotent, weak medial idempotent, quasi-adequate transversal

\section{Introduction and Preliminaries}

Since Blyth and McFadden in [3] introduced the concept of medial idempotent and inverse transversals respectively for a regular semigroup, much attention has been paid to thoes classes of regular semigroups and many papers have been devoted to the theme of describing regular semigroups with medial idempotent (see [2], [7], [12] and its references) and to that of describing regular semigroup with an inverse transversal (see [1], [16] and its references).

Recall from [9] that an idempotent $u$ of an abundant semigroup with a set $E$ of idempotents is called weak medial idempotent if for any $e \in E$, eue $=e$. A weak medial idempotent $u$ is called a weak normal idempotent if $u S u$ is a

Received: April 25, 2013

(c) 2013 Academic Publications, Ltd. url: www.acadpubl.eu 
adequate semigroup. In [15], a weak medial idempotent $u$ is called a quasimedial idempotent for a semigroup $S$ if $u S u$ is a quasi-adequate semigroup, which is the generalization of a weak normal idempotent. The construction of an abundant semigroup with a quasi-medial idempotent was given.

In this paper, some properties for quasi-medial idempotents are obtained. Then it is concerned with the relationship between quasi-medial idempotent and quasi-adequate transversal. We show that if $S$ is a regular semigroup with quasi-medial idempotents $u$ and $w$, then $u S w(u S u, w S w)$ is a quasi-ideal orthodox transversal (see [4]), which is a generalization of inverse transversal. As considering that the class of all abundant semigroups is an important class of generalized regular semigroups, we denote ourself to studying the relationship between quasi-medial idempotent and quasi-ideal quasi-adequate transversal (see [14]), which is an analogous concept of quasi-ideal orthodox transversal in the class of abundant semigroups. Interestingly, if $S$ is an abundant semigroup with quasi-medial idempotents $u$ and $w$ then $u S w(u S u, w S w)$ is a quasi-ideal quasi-adequate transversal for $S$. At last, we consider that in some case an idempotent of an abundant semigroup with a quasi-ideal quasi-adequate transversal can be a quasi-medial idempotent.

The relations $\mathcal{L}^{*}$ and $\mathcal{R}^{*}$ on a semigroup $S$ are generalization of the familiar Green's relations $\mathcal{L}$ and $\mathcal{R}$. Two elements $a$ and $b$ in $S$ are said to be $\mathcal{L}^{*}$-related if and only if they are $\mathcal{L}$-related in some oersemigroup of $S$. The relation $\mathcal{R}^{*}$ is defined dually. A semigroup $S$ is called abundant [8] if each $\mathcal{L}^{*}$-class and $\mathcal{R}^{*}$-class contains an idempotent. An abundant semigroup $S$ is called quasi-adequate [6] if its idempotents form a subsemigroup. Quasi-adequate semigroups are analogues of orthodox semigroups in the range of abundant semigroups. An adequate semigroup is a quasi-adequate semigroup in which the idempotents commute. The class of all abundant semigroups is an important class of generalized regular semigroups. Many authors have investigated various types of abundant semigroups.

The reader is referred to [10], [6] and [8] for all the notation and terminology not defined in this paper.

We shall list some basic results which are used in the sequel. The following lemma is due to Fountain [8] which providing us an alternative description for $\mathcal{L}^{*}\left(\mathcal{R}^{*}\right)$.

Lemma 1.1. (see [8]) Let $S$ be a semigroup, $a \in S$ and e be an idempotent of $S$. Then the following conditions are equivalent:

(1) $a \mathcal{L}^{*} e\left(a \mathcal{R}^{*} e\right)$;

(2) $a=a e(e a=a)$ and for all $x, y \in S^{1}$, ax $=a y(x a=y a) \Rightarrow e x=$ ey $(x e=y e)$. 
Let $S$ be an abundant semigroup and $a \in S$. Denote by $a^{*}\left(a^{+}\right)$a typical idempotent in $\mathcal{L}^{*}$-class ( $\mathcal{R}^{*}$-class) of $S$ containing $a$. The next lemma, is due to El-Qallali [5], will be used frequently in the sequel.

Lemma 1.2. (see [5]) Let $S$ be an abundant semigroup with the set of idempotents $E$ and $x, y \in S$. If there exist $e, f \in E$ such that $x=$ eyf and e $\mathcal{L} y^{+}, f \mathcal{R} y^{*}$ for some $y^{+}, y^{*} \in E$, then $e \mathcal{R}^{*} x$ and $f \mathcal{L}^{*} x$.

Lemma 1.3. (see [9]) Let $S$ be an abundant semigroup with a set $E$ of idempotents and $u$ be a weak medial idempotent of $S$. Then for any $x \in S$ and $e \in E$,

(1) ие, еи, иеи $\in E$;

(2) $x^{+} u \mathcal{R}^{*} x u, u x^{*} \mathcal{L}^{*} u x$;

(3) $u x^{+} u \mathcal{R}^{*} u x u \mathcal{L}^{*} u x^{*} u$.

\section{Quasi-Medial Idempotent}

In this section, unless otherwise stated, we always suppose that $S$ is an abundant semigroup with the set $E$ of idempotents.

Lemma 2.1. Let $u$ be a weak medial idempotent for $S$ and $x \in S$. Then

(1) $x=x^{+} u x=x u x^{*}$;

(2) $x u \mathcal{R} x \mathcal{L} u x$.

Proof. (1) $x=x^{+} x=x^{+} u x^{+} x=x^{+} u x$ and $x=x x^{*}=x x^{*} u x^{*}=x u x^{*}$.

(2) It follows (1) immediately.

Let $U$ be an abundant subsemigroup of $S . \quad U$ is called a left (right) *subsemigroup if for all $a \in U$, there exist $e \in U \cap E$ such that $a \mathcal{L}^{*}(S) e$ $\left(a \mathcal{R}^{*}(S) e\right)$. If $U$ is both a left and a right $*$-subsemigroup, then we call it a *-subsemigroup.

Lemma 2.2. Let $u$ be a weak medial idempotent for $S$ and $e \in E$. Then (1) $u S(S u, u S u)$ is a $*$-subsemigroup;

(2) $E(e S) e \subseteq E(e E(S e) \subseteq E)$.

Proof. (1) Let $x \in u S$. Then by Lemma 1.3 and $2.1, x^{+} u \mathcal{R}^{*} x u \mathcal{R} x$ and $u x^{*} \mathcal{L}^{*} u x \mathcal{L}^{*} x$. Hence $u x^{+} u \mathcal{R}^{*} u x=x$ and $u x^{*} \mathcal{L}^{*} x$ which together with $u x^{+} u, u x^{*} \in E \cap u S$ implies that $u S$ is a $*$-subsemigroup.

(2) Let $x \in E(e S)$. Then $(x e)^{2}=x^{2} e=x e$, whence $E(e S) e \subseteq E$. 
Proposition 2.3. Let $u$ and $w$ be weak medial idempotents for $S$. Then $w S w \cong u S u$.

Proof. Define a map $\phi: w S w \rightarrow u S u$ by $w x w \mapsto u w x w u$. Obviously, $\phi$ is fine. Suppose that $\phi(w x w)=\phi(w y w)$. Then uwxwu $=u w y w u$ implies $w x w=w u w x w u w=w u w y w u w=w y w$. It meas $\phi$ is injective. On the other hand, $\phi(w u x u w)=u w u x u w u=u x u$. Hence by $\phi(w x w y w)=u w x w y w u=$ $u w x w u w y w u=\phi(w x w) \phi(w y w), \phi$ is a isomorphism from $w S w$ onto $u S u$.

We have shown that if $u$ is a weak medial idempotent for $S$ then $u S$ is a *-subsemigroup of $S$. In fact, if $u$ is a quasi-medial idempotent then there are more exciting results.

Lemma 2.4. Let $u$ be a quasi-medial idempotent for $S$. Then $E(u S)=$ $u E(E(S u)=E u, E(u S u)=u E u)$ is a band.

Proof. (1) Let $e, f \in E(u S)$. Since $u e u, u f u \in u E u$,

$$
\begin{aligned}
(e f)^{2} & =(\text { ueuf })^{2}=\text { ueufueufuf } \\
& =(\text { ueufu })^{2} f=\text { ueufuf }=\text { ef }
\end{aligned}
$$

It is trivial to check that $E(u S) \subseteq u E$. Hence $E(u S)=u E$ is a band.

Lemma 2.5. Let $u$ be a weak medial idempotent for $S$. Then the following statements are equivalent:

(1) $u$ is a quasi-medial idempotent;

(2) For any $e \in E, E(e S)$ is a band;

(3) For any $e \in E, E(S e)$ is a band;

(4) For any $e \in E$, eSe is quasi-adequate *-subsemigroup;

(5) $u S$ is a quasi-adequate *-subsemigroup;

(6) $S u$ is a quasi-adequate *-subsemigroup;.

Proof. $(1) \Rightarrow(2)$ Let $x, y \in E(e S)$. Since $x e, y e \in E$,

$$
\begin{aligned}
(x y)^{2} & =(\text { exey })^{2}=[(\text { eue }) x(\text { eue }) y]^{2} \\
& =\text { eu }(\text { xe }) u(\text { ye }) u(x e) u(\text { ye }) y=e u(x e) u(\text { ye }) u(x e) u(\text { ye }) u e y \\
& =\text { eu }\left[(\text { uxeu }(\text { uyeu })]^{2} y=e u(\text { uxeu })(\text { uyeu }) y\right. \\
& =(\text { eue } x(\text { eue }) y=\text { exey }=x y
\end{aligned}
$$

Hence $E(e S)$ is a band.

$(2) \Rightarrow(3)$ Let $x, y \in E(S e)$. As $e x, e y \in E$,

$$
(x y)^{2}=x \text { exeyexey }=x(\text { exey })^{2}=x e x e y=x y \text {, i.e } x y \in E(S e) .
$$


Hence $E(S e)$ is a band.

$(3) \Rightarrow(4)$ Let $x, y \in E(e S e)$. By the similar argument before, $E(e S e)$ is a band. Let $x \in S$. Then by $e x=x, e x^{+}=x^{+}$. It follows that $e x^{+} e x^{+}=x^{+}$ and $x^{+} e x^{+} e=e x^{+} e$. Hence $e x^{+} e \in E(e S e)$ and $x \mathcal{R}^{*} x^{+} \mathcal{R} e x^{+} e$. Dually, $x \mathcal{L}^{*} x^{*} \mathcal{L} e x^{*} e \in E(e S e)$. Therefore $e S e$ is a quasi-adequate $*$-subsemigroup.

$(4) \Rightarrow(5)$ As $e$ is an arbitrary idempotent of $S, u S u$ is quasi-adequate. Then $u E u$ is a band. Let $g, h \in u E$. Then $(g h)^{2}=(u g u h)^{2}=(u g u h u)^{2} h=g h$. Hence $E(u S)$ is a band. By Lemma 2.2, $u S$ is a quasi-adequate $*$-subsemigroup.

$(5) \Rightarrow(6)$ Let $g, h \in E u$. Then $(g h)^{2}=(g u h u)^{2}=g(u g u h)^{2}=g u g u h=g h$ since $E(u S)=u E$ is a band. It means $E(S u)=E u$ is a band. Therefore $S u$ is a quasi-adequate $*$-subsemigroup.

(6) $\Rightarrow(1)$ Let $g, h \in u E u$. Then $(g h)^{2}=(g u h u)^{2}=g u h u=g h$ since $E(S u)=E u$ is a band. Hence $u$ is a quasi-medial idempotent.

In what follows, we shall show that for any quasi-medial idempotents $u$ and $w$ for $S, u w$ is also a quasi-medial idempotent for $S$ and in this case the set of quasi-medial idempotents is a regular subband of $S$.

Lemma 2.6. Let $w$ and $u$ be weak medial idempotents for $S$. Then

(1) For any $e \in E$, uew, euwe $\in E$;

(2) For any $e \in E$, ew $\mathcal{L}$ uew $\mathcal{R}$ ue;

(3) For any $x \in S, x^{*} w \mathcal{L} u x^{*} w \mathcal{L}^{*} u x w \mathcal{R}^{*} u x^{+} w \mathcal{R} u x^{+}$;

(4) $u S w$ is a $*$-subsemigroup.

Proof. (1) Since ew, we $\in E,(u e w)^{2}=u(e w) u(e w)=u e w$ and euweuwe $=$ euwe. Hence uew, euwe $\in E$.

(2) By Lemma 2.1, ew $\mathcal{R}$ e $\mathcal{L}$ ue. Since $\mathcal{R}$ is left congruence, uew $\mathcal{R}$ ue. Dually, ew $\mathcal{L}$ uew.

(3) Since $u x^{*} \mathcal{L}^{*} u x$ and $x w \mathcal{R}^{*} x^{+} w, u x^{*} w \mathcal{L}^{*} u x w \mathcal{R}^{*} u x^{+} w$. On the other hand, from $x^{*} w, u x^{+} \in E$, follows that $x^{*} w \mathcal{L} u x^{*} w$ and $u x^{+} \mathcal{R} u x^{+} w$.

(4) Follows from (3) immediately.

Lemma 2.7. Let $u$ and $w$ be quasi-medial idempotents for $S$. Then

(1) $u E w=u E \cap E w$ is a band;

(2) uwEuw is a band;

(3) $u S w$ is a quasi-adequate $*$-subsemigroup with a band $u E w$.

Proof. (1) As $u E w \subseteq E, u E w \subseteq u E \cap E w$. Obviously, $u E \cap E w \subseteq u E w$. Hence By Lemma 2.4, $u E w=u E \cap E w$ is a band. 
(2) By (1) and its dual, $u w E u w \subseteq u E w \subseteq E$. For any $e, f \in E$,

$$
\begin{aligned}
(\text { uweuwfuw })^{2} & =\text { uweuwfuweuwfuw } \\
& =u(\text { weuwfuw })^{2}=\text { uweuwfuw }
\end{aligned}
$$

as $w e u w, w f u w \in E$. Hence $u w E u w$ is a band.

(3) Follows from (1) and Lemma 2.6.

Proposition 2.8. Let $u$ and $w$ be quasi-medial idempotens for $S$. Then $u w$ is also a quasi-medial idempotent.

Proof. Let $e \in E$. Then by Lemma 2.6, e $\mathcal{R}$ eu $\mathcal{L}$ weu $\mathcal{R}$ we. It follows that e $\mathcal{R}$ eu $\mathcal{R}$ euwe $\mathcal{L}$ we $\mathcal{L}$, whence $e \mathcal{R}$ euwe $\mathcal{L}$ e. Hence $e=$ euwe, which together with Lemma 2.7(2) implies that $u w$ is a quasi-medial idempotent.

Theorem 2.9. Let $S$ be an abundant semigroup with a quasi-medial idempotent $u$. Then the set $U$ of quasi-medial idempotents is a regular subband of $S$.

Proof. Obviously, $U$ is not empty. Let $v, w \in U$. Then $u v w=u w(u v w) u w=$ $u w$ as $u v w$ is a quasi-medial idempotent for $S$. Hence $U$ is a regular subband of $S$.

Let $S$ be an abundant semigroup with the set of idempotents $E$. Denote by $\bar{E}$ the subsemgroup of $S$ generated by $E$. From [11] follows that an idempotent $u$ of $S$ is called medial idempotent if for any $x \in \bar{E}, x e x=x$.

Now we suppose that $u$ and $w$ in Proposition 2.8 and Theorem 2.9 are medial idempotents. Then

Corollary 2.10. Let $u$ and $w$ be medial idempotens for $S$. Then $u w$ is also a medial idempotent.

Proof. Let $x \in \bar{E}$. Since $x u x=x, x u \in E$ and so $x u w \in E$. By Lemma 2.1, $x \mathcal{R}^{*} x u \mathcal{R} x u w$. It meas $x u w x=x$. Hence $u w$ is a medial idempotent.

Corollary 2.11. Let $S$ be an abundant semigroup with a medial idempotent $u$. Then the set $U$ of medial idempotents is a regular subband of $S$. 


\section{Relationship between Quasi-Medial Idempotents and Quasi-Adequate Transversals}

In this section, it shall be concerned with the relationship between quasi-medial idempotents and quasi-ideal transversals. In fact, let $S$ be a semigroup with quasi-medial idempotents $u$ and $w$. Firstly, we will show that if $S$ is regular then $u S w(u S u, w S w)$ is a quasi-ideal orthodox transversal for $S$ and that if $S$ is an abundant semigroup then $u S w(u S u, w S w)$ is a quasi-ideal quasi-adequate transversal for $S$. Next, let $S$ be an abundant semigroup with a quasi-ideal quasi-adequate transversal $S^{\circ}$. We shall consider in which case an idempotent of $S$ is a quasi-medial idempotent.

For any subsemigroup $T$ of a semigroup $S$, let $V_{T}(x)=V(x) \cap T$ where $x \in S$. Moreover, $T$ is a quasi-ideal of $S$ if $T S T \subseteq T$.

Lemma 3.1. Let $S$ be an abundant semigroup with weak medial idempotents $u$ and $w$. Then

(1) For any $x^{\prime} \in V(S), x u x^{\prime}=x x^{\prime}$ and $x^{\prime} u x=x^{\prime} x$;

(2) $(\forall x \in S) V(x) \neq \emptyset \Rightarrow u V(x) w=V_{u S w}(x)=V_{u S w}(u x w)$

(3) $(\forall x \in S) V(x) \neq \emptyset \Rightarrow V_{u S w}(x)=V_{u S w}(w x)=V_{u S w}(x u)=V_{u S w}(w x u)$;

(4) $(\forall x \in S) V(x) \neq \emptyset \Rightarrow V_{u S w}(x)=V_{u S u}(x) x V_{w S w}(x)$.

Proof. (1) Obviously, $x^{\prime} x, x x^{\prime} \in E$. Hence $x u x^{\prime}=x x^{\prime} x u x^{\prime} x x^{\prime}=x x^{\prime} x=x$ and $x^{\prime} u x^{\prime}=x^{\prime} x x^{\prime} u x^{\prime} x x^{\prime}=x^{\prime} x x^{\prime}=x^{\prime}$.

(2) Let $x^{\prime} \in V(x)$. Then $u x^{\prime} w x u x^{\prime} w=u x^{\prime} x x^{\prime} w=u x^{\prime} w$ and $w x u x^{\prime} w x u=$ $u x x^{\prime} x w=w x u$. It means $u V(x) w \subseteq V_{u S w}(x)$, i.e $V_{u S w}(x) \neq \emptyset$. Obviously, $V_{u S w}(x) \subseteq u V(x) w$. Therefore $u V(x) w=V_{u S w}(x)$.

Next we shall prove that $V_{u S w}(x)=V_{u S w}(u x w)$. Let $y \in V_{u S u}(x)$. Take $x^{+}=x y$ and $x^{*}=y x$. Then by Lemma 2.1,

$$
\begin{aligned}
x y x & =\left(x^{+} u x w x^{*}\right) y\left(x^{+} u x w x^{*}\right) \\
& =x^{+} u x w(y x) y(x y) u x w x^{*} \quad\left(\text { since } x^{+}=x y, x^{*}=y x\right) \\
& =x^{+} u x w y u x w x^{*} \quad\left(\text { since } y \in V_{u S w}(x)\right) \\
& =x^{+} u x y x w x^{*} \\
& =x^{+} u x w x^{*}=x
\end{aligned}
$$

and $y=y x y=y x^{+} u x w x^{*} y=y x y u x w y x y=y u x w y$. Hence $V_{u S u}(x) \subseteq$ $V_{u S u}(u x u)$. Conversely, let $y \in V_{u S w}(u x w)$. Notice that

$$
x^{+} w \mathcal{L} u x^{+} w \mathcal{R}^{*} u x w \mathcal{R}^{*} u x w y \mathcal{L}^{*} y
$$

and that

$$
u x^{*} \mathcal{R} u x^{*} w \mathcal{L}^{*} u x w \mathcal{L}^{*} \text { yuxw } \mathcal{R}^{*} y .
$$


Then $x^{+} w(u x w y) \mathcal{L}^{*} y \mathcal{R}^{*}(y u x w) u x^{*}$. By commuting,

$$
\begin{aligned}
x y x & =\left[x^{+} w(u x w) u x^{*}\right] y\left[x^{+} w(u x w) u x^{*}\right] \\
& =\left[x^{+} w(u x w) y(u x w) u x^{*}\right] y\left[x^{+} w(u x w) y(u x w) u x^{*}\right] \\
& =x^{+} w(u x w)\left[(y u x w) u x^{*} y x^{+} w(u x w y)\right](u x w) u x^{*} \\
& =x^{+} w(u x w) y(u x w) u x^{*} \\
& =x^{+} w u x w u x^{*}=x
\end{aligned}
$$

and

$$
\begin{aligned}
y x y & =y\left[x^{+} w(u x w) u x^{*}\right] y \\
& =y x^{+} w[(u x w) y(u x w) y(u x w)] u x^{*} y \\
& =y\left(x^{+} w u x w y\right)(u x w)\left(y u x w u x^{*}\right) y \\
& =y(u x w) y=y .
\end{aligned}
$$

Hence $V_{u S w}(u x w) \subseteq V_{u S w}(x)$.

(3) By $(2), V_{u S w}(x) \neq \emptyset$. Here we just prove that $V_{u S w}(x)=V_{u S w}(w x)$. The remainder part can be proved by the similar arguments. In fact, we suppose that $x^{\prime} \in V_{u S w}(x)$. Then $x^{\prime}(w x) x^{\prime}=x^{\prime} x x^{\prime}=x^{\prime}$ and $w x x^{\prime} w x=w x x^{\prime} x=w x$, whence $V_{u S w}(x) \subseteq V_{u S w}(w x)$. Conversely, let $y \in V_{u S w}(w x)$. Then $y x y=$ $(y w) x y=y(w x) y=y$ and $x y x=x^{+} w x y w x w x^{*}=x^{+} w x w x^{*}=x$. It means $V_{u S w}(w x) \subseteq V_{u S w}(x)$.

(4) Let $y \in V_{u S u}(x)$ and $z \in V_{w S w}(x)$. Then by computing, $(y x z) x(y x z)=$ $y(x z x) y x z=y x y x z=y x z$ and $x(y x z) x=(x y x) z x=x z x=x$. It follows that $V_{u S u}(x) x V_{u S w}(x) \subseteq V_{u S w}(x)$. Conversely, let $a \in V_{u S w}(x)$. Then for any $x^{\prime} \in V(x), a=a x a=a x\left(u x^{\prime} u\right) x\left(w x^{\prime} w\right) a=\left(a x x^{\prime} u\right) x\left(w x^{\prime} x a\right)$. Notice that $\left(a x x^{\prime} u\right) x\left(a x x^{\prime} u\right)=a x a x x^{\prime} u=a x x^{\prime} u$ and $x\left(a x x^{\prime} u\right) x=x a x=x$. Hence by $u a=u, a x x^{\prime} u \in V_{u S u}(x)$. Similarly, we have $w x^{\prime} x a \in V_{w S w}(x)$. Therefore $V_{u S w}(x) \subseteq V_{u S u}(x) x V_{u S w}(x)$.

As we all know, a regular semigroup $S$ is orthodox if and only if

$$
(\forall a, b \in S) V(a) \cap V(b) \neq \emptyset \Rightarrow V(a)=V(b) .
$$

Here we shall show that a regular semigroup with two quasi-medial idempotents have the similar result.

Lemma 3.2. Let $S$ be a regular semigroup with quasi-medial idempotents $u$ and $w$. Then

(1) $(\forall x \in S) V_{u S w}(x) \neq \emptyset$;

(2) $(\forall x, y \in S) V_{u S w}(x) \cap V_{u S w}(y) \neq \emptyset \Rightarrow V_{u S w}(x)=V_{u S w}(y)$;

(3) $(\forall x, y \in S)\{x, y\} \cap u S w \neq \emptyset \Rightarrow V_{u S w}(x) V_{u S w}(y) \subseteq V_{u S w}(y x)$. 
Proof. (1) Let $x^{\prime} \in V(x)$. In view of the proof of Lemma 3.1 (2), $u x^{\prime} w \in$ $V_{u S w}(x)$.

(2) Since $V_{u S w}(x)=V_{u S w}(u x w)$ and $V_{u S w}(y)=V_{u S w}(u y w), V_{u S w}(u x w) \cap$ $V_{u S w}(u y w) \neq \emptyset$. Notice that $u S w$ is orthodox. We have $V_{u S w}(u x w)=V_{u S w}(u y w)$, whence $V_{u S w}(x)=V_{u S w}(y)$.

(3) Suppose that $y \in u S w$. As $u S w$ is an orthodox semigroup, we have

$$
\begin{aligned}
V_{u S w}(x) V_{u S w}(y) & =V_{u S w}(w x) V_{u S w}(y) \\
& =V_{u S w}\left(\text { uwxw) } V_{u S u}(y)\right. \\
& \subseteq V_{u S w}(\text { yuwxw) } \\
& =V_{u S w}(\text { uywuwxw) } \\
& =V_{u S w}(\text { uyxw })=V_{u S u}(y x) .
\end{aligned}
$$

Recall from [4] that a subsemigroup $S^{\circ}$ of a regular semigroup $S$ is called an orthodox transversal for $S$ if

$(O 1)(\forall x \in S) \quad V_{S^{\circ}}(x) \neq \emptyset$,

$(O 2)(\forall x, y \in S)\{x, y\} \cap S^{\circ} \neq \emptyset \Rightarrow V_{S^{\circ}}(x) V_{S^{\circ}}(y) \subseteq V_{S^{\circ}}(y x)$.

Then by Lemma 2.7 and 3.2, we have

Theorem 3.3. Let $S$ be a regular semigroup with quasi-medial idempotents $u$ and $w$. Then $u S w$ is a quasi-ideal orthodox transversal for $S$. In particular, $u S u$ and $w S w$ are also quasi-ideal orthodox transversals for $S$.

For convenience, we list some notaion about quasi-adequate transversals as follows [13]. The reader is referred to [13] and [14] for all the notation, terminology and some results not mentioned in this section.

Let $S$ be an abundant semigroup and $S^{\circ}$ an abundant subsemigroup with the set of idempotents $E^{\circ}$. $S^{\circ}$ is called an abundant transversal for $S$ if for any $x \in S$, there exist $x^{\circ} \in S^{\circ}, e, f \in E$ such that $x=e x^{\circ} f$, where $e \mathcal{L}^{*} x^{\circ+}$, $f \mathcal{R}^{*} x^{\circ *}$ for some $x^{\circ+}, x^{\circ *} \in E^{\circ}$. In this case, let

$$
\begin{gathered}
C_{S^{\circ}}(x)=\left\{x^{\circ} \in S^{\circ} \mid x=e x^{\circ} f, e \mathcal{L} x^{\circ+}, f \mathcal{R} x^{\circ *}, x^{\circ+}, x^{\circ *} \in E^{\circ}\right\}, \\
I_{x}=\left\{e \in E \mid\left(\exists x^{\circ} \in C_{S^{\circ}}(x)\right) x=e x^{\circ} f, e \mathcal{L} x^{\circ+}, f \mathcal{R} x^{\circ *}, x^{\circ+}, x^{\circ *} \in E^{\circ}\right\}, \\
\Lambda_{x}=\left\{f \in E \mid\left(\exists x^{\circ} \in C_{S^{\circ}}(x)\right) x=e x^{\circ} f, e \mathcal{L} x^{\circ+}, f \mathcal{R} x^{\circ *}, x^{\circ+}, x^{\circ *} \in E^{\circ}\right\}, \\
I=\bigcup_{x \in S} I_{x}, \quad \Lambda=\bigcup_{x \in S} \Lambda_{x} .
\end{gathered}
$$

$S^{\circ}$ is called a quasi-adequate transversal for $S$ if $S^{\circ}$ is quasi-adequate and $(Q A 1) \quad(\forall x \in S) \quad C_{S^{\circ}}(x) \neq \emptyset$, 
$(Q A 2)(\forall e \in E)\left(\forall g \in E^{\circ}\right)$

$$
C_{S^{\circ}}(e) C_{S^{\circ}}(g) \subseteq C_{S^{\circ}}(g e) \text { and } C_{S^{\circ}}(g) C_{S^{\circ}}(e) \subseteq C_{S^{\circ}}(e g)
$$

A quasi-adequate transversal $S^{\circ}$ is multiplicative if $(M) \quad(\forall x, y \in S) \quad \Lambda_{x} I_{y} \subseteq E^{\circ}$.

A quasi-adequate transversal $S^{\circ}$ is a quasi-ideal quasi-adequate transversal for $S$ if $S^{\circ}$ is a quasi-ideal of $S$ (i.e, $S^{\circ} S S^{\circ} \subseteq S^{\circ}$ ).

In what follows, unless otherwise stated, we always suppose that $S$ is an abundant semigroup with the set $E$ of idempotents.

Lemma 3.4. Let $u$ and $w$ be quasi-medial idempotents for S. For any $x \in S$,

(1) $u x w \in C_{u S w}(x)$;

(2) $x^{+} w \in I_{x}$ and $u x^{*} \in \Lambda_{x}$.

Proof. (1), (2) Obviously, $x=x^{+} w u x w u x^{*}$ since $w u$ is a quasi-medial idempotent. Notice that $x^{+} w, u x^{*} \in E$. Then by Lemma 1.3 and 2.1,

$$
x^{+} w \mathcal{L} u x^{+} w \mathcal{R}^{*} u x w \text { and } u x^{*} \mathcal{R} u x^{*} w \mathcal{L}^{*} u x w .
$$

Hence $u x w \in C_{u S w}(x)$ and $x^{+} w \in I_{x}, u x^{*} \in \Lambda_{x}$.

Lemma 3.5. Let $u$ and $w$ be quasi-medial idempotents for $S$. Then

(1) $I=E w$ and $\Lambda=u E$;

(2) $I \cap \Lambda=E w \cap u E=u E w$;

(3) $\Lambda I \subseteq u S w$.

Proof. (1) By Lemma 3.4, $u S w$ is a abundant transversal for $S$. Then for any $g \in I$, there exists $x \in S$ and $x^{\circ} \in C_{u S w}(x)$ such that $g \mathcal{L} x^{\circ+}$ for some $x^{\circ+} \in u E w$. It follows that $g=g x^{\circ+}=g x^{\circ+} w=g w \in E w$. On the other hand, let $h \in E w$. Then $h=h w \mathcal{L} u h w \in u E w$. It means $h \in I_{h} \subseteq I$. Hence $I=E w$. The remained proof is a dual.

(2), (3) It is trivial.

Let $S$ be a quasi-adequate semigroup with a band $B$. For $e \in B$, denote by $E(e)$ the $\mathcal{J}$-class of $B$ containing $e$. Define a relation $\delta$ on $S$ by: for $a, b \in S$,

$$
a \delta b \Leftrightarrow E\left(a^{+}\right) a E\left(a^{*}\right)=E\left(b^{+}\right) b E\left(b^{*}\right) \text { for some } a^{+}, a^{*}, b^{+}, b^{*} .
$$

It follows from [6] that $\delta$ is an equivalence relation which contained in any adequate congruence on $S$. In particular, if $S$ is an orthodox semigroup, then $\delta$ is the least inverse congruence on $S$. 
Lemma 3.6. Let $u$ and $w$ be quasi-medial idempotents for $S$. Then for any $e \in E$ and $g \in u E w$,

(1) $C_{u S w}(e)=\delta_{\text {uew }} \subseteq u E w$;

(2) $C_{u S w}(e) C_{u S w}(g) \subseteq C_{u S w}(g e)$ and $C_{u S w}(g) C_{u S w}(e) \subseteq C_{u S w}(e g)$.

Proof. (1) Let $e^{\circ} \in C_{u S u}(e)$. Then there exists $i_{e} \in I_{e}$ and $\lambda_{e} \in \Lambda_{e}$ such that $e=i_{e} e^{\circ} \lambda_{e}$ and $i_{e} \mathcal{L} e^{\circ+}, \lambda_{e} \mathcal{R} e^{\circ *}$ for some $e^{\circ+}, e^{\circ *} \in u E w$. It is easy to check that $e^{\circ}=e^{\circ+} e e^{\circ *}=\left(e^{\circ+} e w\right) u e w\left(u e e^{\circ *}\right)$ as uw and $w u$ are quasimedial idempotents. By Lemma 1.2, $i_{e} \mathcal{R}$ e $\mathcal{L} \lambda_{e}$. Hence ew $\mathcal{R} i_{e} \mathcal{L} e^{o+}$ and ue $\mathcal{L} \lambda_{e} \mathcal{R} e^{o *}$. Then uew $\mathcal{L}$ ew $\mathcal{L}^{*} e^{\circ+}$ ew and uew $\mathcal{R}$ ue $\mathcal{R}^{*}$ uee $e^{\circ+}$. Obviously, $e^{\circ+} e w, u e e^{\circ+} \in u E w$. Therefore $e^{\circ+} e w, u e e^{\circ+} \in E(u e w)$ and so $e^{\circ} \in \delta_{\text {uew }}$.

Conversely, let $\bar{e} \in \delta_{\text {uew }}$. Obviously, $\delta_{\text {uew }} \subseteq u E w$. Then $\bar{e} \in u E w$ and uewe $\mathcal{L} \bar{e} \mathcal{R}$ éuew. Notice that $\bar{e}, e w \in I$. We have $\bar{e} \delta(I)$ ew. It follows that ew $\mathcal{R}$ ewe $\mathcal{L} \bar{e}$. Dually, ue $\mathcal{L} \bar{e} u e \mathcal{R} \bar{e}$. Hence ewe $\mathcal{L}$ uewe $\mathcal{L} \bar{e}$ and $\bar{e}$ uew $\mathcal{R}$ e ue $\mathcal{R} \bar{e}$. On the other hand, we have $e=e w u e=e w($ uew $) \bar{e}($ uew $)$ ue $=$ ewēue. Therefore $\bar{e} \in C_{u S u}(e)$.

(2)As $g=u g w$ and euwe $=e$, ugew $=$ gwew $=g w($ uwew $)($ uew $)$ and $g w($ uew $)($ uwew $)=$ gwuew $=$ guew. For any $x, y \in u E w, x \delta(u E w) y$ if and only $x \delta y$. Hence

$$
\text { uewg } \delta \text { guew }=g w(\text { uew })(\text { uwew }) \delta g w(\text { uew })(\text { uew })=\text { ugew. }
$$

It follows that $\delta_{\text {uew }} \delta_{g} \subseteq \delta_{\text {uewg }}=\delta_{\text {ugew }}$, whence $C_{u S u}(e) C_{u S u}(g) \subseteq C_{u S u}(g e)$. Similarly, $C_{u S u}(g) C_{u S u}(e) \subseteq C_{u S u}(e g)$.

Theorem 3.7. Let $S$ be an abundant semigroup with quasi-medial idempotents $u$ and $w$. Then $u S w$ is a quasi-ideal quasi-adequate transversal for $S$. In particular, $u S u$ and $w S w$ are also quasi-ideal quasi-adequate transversals for $S$.

Proof. By Lemma 3.4 and 3.6.

Corollary 3.8. Let $S$ be a abundant semigroup with medial idempotents $u$ and $w$. Then $u S w$ is a multiplicative quasi-adequate transversal for $S$. In particular, $u S u$ and $w S w$ are also multiplicative quasi-adequate transversals for $S$.

Proof. Let $e, f \in E$. Then $e w \in E w, u f \in u E$. By Corollary 2.10, $(u f e w)^{2}=u($ fewufe $) w=u f e w$. It means $\Lambda I \subseteq u E w$. Hence by Theorem 3.7, $u S w, u S u$ and $w S w$ are multiplicative quasi-adequate transversals. 
Lemma 3.9. Suppose that $S^{\circ}$ is a quasi-ideal quasi-adequate transversal for $S$. Let $x \in S$ and $i_{x} \in I_{x}, x^{\circ} \in C_{S^{\circ}}(x), \lambda_{x} \in \Lambda_{x}$ such that $x=i_{x} x^{\circ} \lambda_{x}$ and $i_{x} \mathcal{L} x^{\circ+}, \lambda_{x} \mathcal{R} x^{\circ *}$. If $x^{\circ} \in E^{\circ}$ and $u \in E^{\circ}$ is an identity of $E^{\circ}$, then $\lambda_{x} u i_{x}=x^{\circ *} x^{\circ+}$.

Proof. In fact, since $u$ is an identity of $E^{\circ}$,

$$
\lambda_{x} u \lambda_{x}=\lambda_{x} u\left(x^{\circ *} \lambda_{x}\right)=\lambda_{x}\left(u x^{\circ *}\right) \lambda_{x}=\lambda_{x}\left(x^{\circ *} \lambda_{x}\right)=\lambda_{x} .
$$

It follows that $\lambda_{x} u \in E$. It is trivial to check that $\lambda_{x} u \mathcal{R} \lambda_{x}$. Similarly, $u i_{x} \mathcal{L} i_{x}$. Notice that $x^{\circ} \in E^{\circ}$. We have $x^{\circ *} \mathcal{R} x^{\circ *} x^{\circ+} \mathcal{L} x^{\circ+}$. Then $u i_{x} \mathcal{L} x^{\circ *} x^{\circ+} \mathcal{R} \lambda_{x} u$. From $\lambda_{x} u, u i_{x} \in E^{\circ}$ follows that $\lambda_{x} u \delta\left(E^{\circ}\right) u i_{x}$. It means $\lambda_{x} u \mathcal{R} \lambda_{x} u i_{x} \mathcal{L} u i_{x}$. Hence $\lambda_{e} u i_{e} \mathcal{H}^{*} e^{\circ *} e^{\circ+}$, which together with $\lambda_{x} u i_{x}, x^{\circ *} x^{\circ+} \in E^{\circ}$ implies that $\lambda_{x} u i_{x}=x^{\circ *} x^{\circ+}$.

Proposition 3.10. Let $S^{\circ}$ be a quasi-ideal quasi-adequate transversal for $S$. If for any $e \in E, C_{S^{\circ}}(e) \subseteq E^{\circ}$ and $u$ is an identity of $E^{\circ}$, then $u$ is a quasi-medial idempotent for $S$.

Proof. Let $i_{e} \in I_{e}, e^{\circ} \in C_{S^{\circ}}(e)$ and $\lambda_{e} \in \Lambda_{e}$ be such that $e=i_{e} e^{\circ} \lambda_{e}$ and $i_{e} \mathcal{L} e^{\circ+}, \lambda_{e} \mathcal{R} e^{0 *}$. Then eue $=i_{e} e^{\circ} \lambda_{e} u i_{e} e^{\circ} \lambda_{e}=i_{e} e^{\circ} e^{\circ *} e^{\circ+} e^{\circ} \lambda_{e}=i_{e} e^{\circ} \lambda_{e}=e$. On the other hand, $u E u \subseteq S^{\circ} \cap E=E^{\circ}$ as $S^{\circ}$ is a quasi-ideal. It follows that $(u E u)(u E u) \subseteq E^{\circ} \cap u S u \subseteq u E u$. Hence $u E u$ is a band and so $u$ is a quasi-medial idempotent for $S$.

Proposition 3.11. Let $S^{\circ}$ be a multiplicative quasi-adequate transversal for $S$. If $u$ is an identity of $E^{\circ}$, then $u$ is a medial idempotent for $S$.

Proof. Let $e, f \in E$. Then there exists $i_{e} \in I_{e}, i_{f} \in I_{f}, e^{\circ} \in C_{S^{\circ}}(e f), f^{\circ} \in$ $C_{S^{\circ}}(f)$ and $\lambda_{e} \in \Lambda_{e}, \lambda_{f} \in \Lambda_{f}$ such that $e=i_{e} e^{\circ} \lambda_{e}, i_{e} \mathcal{L} e^{\circ+}, \lambda_{e} \mathcal{R} e^{\circ *}$ for some $e^{\circ+}, e^{\circ *} \in E^{\circ}$ and $f=i_{f} f^{\circ} \lambda_{f}, i_{f} \mathcal{L} f^{\circ+}, \lambda_{f} \mathcal{R} f^{\circ *}$ for some $f^{\circ+}, f^{\circ *} \in E^{\circ}$. Since $S^{\circ}$ is a multiplicative quasi-adequate transversal for $S, e^{\circ}, f^{\circ} \in E^{\circ}$ by Lemma 2.3 of [14] and $e^{\circ} \lambda_{e} i_{f} f^{\circ} \in C_{S^{\circ}}(e f)$ by Theorem 2.9 of [14]. Notice that $\lambda_{e} i_{f} \in E^{\circ}$. We have $e^{\circ} \lambda_{e} i_{f} f^{\circ} \in E^{\circ}$. On the other hand, since $e^{\circ+}\left(e^{\circ} \lambda_{e} i_{f} f^{\circ}\right)=$ $e^{\circ} \lambda_{e} i_{f} f^{\circ}, e^{\circ+}\left(e^{\circ} \lambda_{e} i_{f} f^{\circ}\right)^{+}=\left(e^{\circ} \lambda_{e} i_{f} f^{\circ}\right)^{+}$for some $\left(e^{\circ} \lambda_{e} i_{f} f^{\circ}\right)^{+} \in E^{\circ}$. Then by $i_{e} \mathcal{L} e^{\circ+}, i_{e}\left(e^{\circ} \lambda_{e} i_{f} f^{\circ}\right)^{+} \mathcal{L}\left(e^{\circ} \lambda_{e} i_{f} f^{\circ}\right)^{+}$. Dually, $\left(e^{\circ} \lambda_{e} i_{f} f^{\circ}\right)^{*} \lambda_{f} \mathcal{R}\left(e^{\circ} \lambda_{e} i_{f} f^{\circ}\right)^{*}$ for some $\left(e^{\circ} \lambda_{e} i_{f} f^{\circ}\right)^{*} \in E^{\circ}$. Obviously,

$$
e f=i_{e} e^{\circ} \lambda_{e} i_{f} f^{\circ} \lambda_{f}=i_{e}\left(e^{\circ} \lambda_{e} i_{f} f^{\circ}\right)^{+}\left(e^{\circ} \lambda_{e} i_{f} f^{\circ}\right)\left(e^{\circ} \lambda_{e} i_{f} f^{\circ}\right)^{*} \lambda_{f} .
$$

Hence in view of the proof of Lemma 3.9,

$$
\left(e^{\circ} \lambda_{e} i_{f} f^{\circ}\right)^{*} \lambda_{f} u i_{e}\left(e^{\circ} \lambda_{e} i_{f} f^{\circ}\right)^{+}=\left(e^{\circ} \lambda_{e} i_{f} f^{\circ}\right)^{*}\left(e^{\circ} \lambda_{e} i_{f} f^{\circ}\right)^{+} .
$$


It follows that efuef $=i_{e}\left(e^{\circ} \lambda_{e} i_{f} f^{\circ}\right) \lambda_{f}=e f$. Therefore by Mathematic Induction, for any $x \in \bar{E}, x u x=x$.

Remark. The converse of Theorem 3.11 is false.

Example. Let $\mathrm{S}$ be a four-element semigroup given by the following Cayley table:

\begin{tabular}{l|llll} 
& e & f & g & a \\
\hline e & e & f & e & a \\
f & f & f & f & a \\
g & g & f & g & a \\
a & a & a & a & f
\end{tabular}

Then $S$ is a quasi-adequate semigroup with a band $E(S)=\{e, f, g\}$ and $f \mathcal{H}^{*} a$, $g \mathcal{L} e$. Obviously, $S$ is a multiplicative quasi-adequate transversal for itself. It is easy to check that $e$ is a medial idempotent for $S$ for all but an identity of $E(S)$.

\section{Acknowledgments}

This research was supported by National Natural Science Foundation of P.R. China (No. 11226050).

\section{References}

[1] T.S. Blyth, R. McFadden, Regular semigroups with a multiplicative inverse transversal, Proc. Roy. Soc. Edinburgh 92A (1982), 253-270.

[2] T.S. Blyth, R. McFadden, On the construction of a class of regular semigroups, J. Algebra, 81(1983), 1-22.

[3] T.S. Blyth, R. McFadden, Regular semigroups with a greastest idempotent, Proc.Roy.Soc.Edinburgh, 91A (1981), 107-122.

[4] J.F. Chen, On regular semigroups with orthodox transversals, Commun. Agebra 27, No. 9 (1999), 4275-4288.

[5] A. El-Qallali, Abundant semigrops with a amultiplicative type A transversl, Semigroup Forum 47 (1993), 327-340.

[6] A. El-Qallali, J.B. Fountain, Quasi-adequate semigroups, Proc. Roy. Soc. Edinburgh, 91A (1981), 91-99. 
[7] A. El-Qallali, On the construction of a class of abundant semigroups, Acta. Math. Hung, 56 (1990), 77-91.

[8] J.B. Fountain, Abundant semigroups, Proc. London Math. Soc. 44, No. 3 (1982), 103-129.

[9] X.J. Guo, The Structure of Abundant Semigroups with a Weak Normal Idempotent, Acta. Math. Sin. , 42 (1999), 683-690.

[10] J.M. Howie, Fundamentals of Semigroup Theory, Clarendon Press, Oxford, 1995.

[11] F.J. Jing, Abundant semigroupss with a medial idempotent, Semigroup Forum, 51 (1995), 247-261.

[12] M. Longanathan, Regular semigroups with a medial idempotent, Semigroup Forum, 36 (1987), 69-74.

[13] X.F. Ni, Abundant semigroups with a multiplicative quasi-adequate transversal, Semigroup Forum, 78 (2009), 34-53.

[14] X.F. Ni, Y.F. Luo, H.Z. Chao, Abundant semigroups with a quasi-ideal quasi-adequate transversal, Acta. Math. Sin., 25 (2009), 1653-1664.

[15] X.F. Ni, Y.F. Luo , abundant semigroup with quasi-medial idempotents, Inter. J. Pure Ap. Mat., 72 (2011), 81-91.

[16] X.L. Tang, Regular semigroups with inverse transversals, Semigroup Forum, 55 (1997), 24-32. 